\title{
Principios e implicaciones del Nuevo Modelo Educativo
}

\author{
Principles and implications \\ of the New Educational Model
}

Federico Ortega Estrada*

\section{RESUMEN}

Este documento analiza los principios pedagógicos que están presentes en los enfoques que se enuncian en el Nuevo Modelo Educativo 2016 (NME) y en la Propuesta Curricular (PC): el aprendizaje profundo, el aprendizaje situado, el aprendizaje significativo y el aprendizaje socioemocional. Se realiza un análisis de las implicaciones de llevar a la práctica dichos enfoques en el marco de la realidad de las escuelas públicas del país. Para ello se recuperan otros ejes de política que se exponen en los documentos, tales como la educación de calidad como un derecho constitucional, la educación inclusiva con equidad y el tema de las barreras de aprendizaje.

Palabras clave: Reforma educativa, propuesta curricular, autonomía escolar, aprendizaje

\begin{abstract}
This paper analyzes the pedagogical principles that are present in the approaches mentioned in the New Education Model 2016 (NME) and in the Curricular Proposal (PC): deep learning, situated learning, meaningful learning and socioemotional learning. Analysis is being performed on the implications of putting into practice such approaches within the framework of the reality of the country's public schools. This involves the recovery of the other political instances which are exposed in the documents, such as quality education as a constitutional right, fair inclusive education and the issue of learning barrierss
\end{abstract}

Key words: Educational reform, curriculum proposal, school autonomy, learning

\section{INTRODUCCIÓN}

Destacan al menos tres enfoques o principios pedagógicos en el Nuevo Modelo Educativo (NME) y en la Propuesta Curricular (PC) que publicó recientemente la Secretaría de Educación Pública (SEP), en el marco del Reforma Educativa. Se trata del aprendizaje profundo, el aprendizaje situado y el aprendizaje significativo y, junto con ello, una perspectiva que busca desarrollar las competencias emocionales y sociales. De manera transversal están presentes: el enfoque del aprendizaje basado en competencias y el tema de los aprendizajes clave.

\footnotetext{
" Instituto Nacional para la Evaluación de la Educación (INEE) en el estado de Chihuahua; fortega77@yahoo.com
} 
Además, se identifican otras categorías como la educación de calidad como un derecho constitucional, la educación inclusiva con equidad y, asociado a estos, el tema de las barreras de aprendizaje.

Para esto se reconoce que las escuelas han dejado de ser el único lugar para aprender, o más bien, ya no ocupan el lugar protagónico que tenían en el proceso de aprendizaje. El NME también se pronuncia contra la memorización de conceptos, hechos y procedimientos, así como contra la saturación de contenidos.

Conforme a lo anterior, el documento enfatiza que el corazón del NME es "el planteamiento pedagógico" el cual se describe tanto en el Modelo como en la Propuesta Curricular. Este planteamiento contiene "[...] la organización y los procesos que tienen lugar en la escuela, las prácticas pedagógicas en el aula y el currículo" (SEP, 2016a: 18).

Para lograr un aprendizaje profundo, situado y significativo se parte de un principio de política pública: ubicar al centro escolar como el eje de todas las acciones de las autoridades educativas, los recursos, apoyos y acompańamientos que la escuela necesita: esto conforme al lema Escuela al Centro.

Este nuevo enfoque de Escuela al Centro rescata el principio de educación inclusiva con equidad y reconoce que para recuperarlo y llevarlo a la práctica "[...] todos los elementos del modelo educativo -currículo, directores, docentes, padres de familia, infraestructura, presupuesto, procesos, flujos de información, entre otros" (SEP, 2016a: 19)- han de responder a este principio. Conforme a este, ocupan el primer lugar la población indígena, las personas con discapacidad y la población en situación vulnerable. De acuerdo con dicho enfoque iniciaré por analizar lo que el NME llama el corazón, es decir, el planteamiento pedagógico y, más adelante, sus implicaciones pedagógicas y de política pública.

La razón de incorporar un nuevo planteamiento pedagógico la expone detalladamente el documento Propuesta Curricular 2016 señalando que el currículo vigente:

- se enfoca más en la enseñanza que en el aprendizaje;

- es muy extenso;

- no da lugar a que los alumnos profundicen en ideas y conceptos;

- desestima las necesidades de aprendizaje de los educandos;

- no ofrece una formación integral; 
- se focaliza en temas académicos;

- es cerrado y poco flexible (SEP, 2016b: 43).

De acuerdo tanto con la PC y con NME la solución está en que en las escuelas se lleve a cabo un aprendizaje más activo, autorregulado, dirigido a metas, situado, colaborativo y que facilite los procesos personales de construcción de significados y conocimientos (SEP, 2016b: 33; SEP, 2016a: 50). Para ello proponen cuatro enfoques pedagógicos: aprendizaje profundo, aprendizaje significativo, aprendizaje situado y aprendizaje socioemocional. En la siguiente parte se revisan los cuatro planteamientos.

\section{PLANTEAMIENTOS PEDAGÓGICOS}

\section{Aprendizaje profundo}

La PC señala que, debido a que no se favorece en los alumnos la profundización de ideas y conceptos, no se desarrollan habilidades cognitivas superiores, ni las competencias requeridas en el siglo XXI. De acuerdo con López y López (2013), el concepto de aprendizaje profundo fue expuesto en la década de los setenta por Marton y Säljö, pero hasta ahora ha tomado fuerza en los debates y planteamientos de los enfoques que deben predominar en los planes de estudio.

Es indudable que propiciar prácticas docentes orientadas a que los alumnos logren el aprendizaje profundo, necesariamente demanda reestructurar o rediseñar "la organización y los procesos que tienen lugar en la escuela, las prácticas pedagógicas en el aula y el currículo" (SEP, 2016a: 18), como el mismo NME establece.

En las condiciones actuales, incorporar los presupuestos y la metodología del aprendizaje profundo, salvo en algunos centros educativos, es prácticamente imposible. En el medio urbano la mayoría de los docentes labora en grupos con 30 o hasta 60 alumnos. Además, hay una saturación de contenidos en los planes de estudio que abordarlos de manera completa se vuelve la preocupación principal de los docentes, no importa que sea de manera superficial; "yo ya voy en la unidad IV, no sé mis alumnos" es la frase típica que se escucha en los pasillos de las escuelas. Las técnicas o estrategias de enseñanza difícilmente van más allá 
del repaso simple, subrayar o contestar preguntas acotadas. En términos de Pozo (1990), estas técnicas se quedan en el nivel de recirculación de la información y, en algunos casos, del procesamiento simple. Los condicionamientos del plan de estudios, del tiempo y del espacio físico no dan para más.

Probablemente en algunas escuelas de educación primaria y secundaria que cuentan con grupos pequeños e instalaciones adecuadas pueden implementar algunas estrategias del aprendizaje profundo. En estas condiciones, puede ser que, si el profesor conoce el enfoque del plan de estudios y cuenta con herramientas metodológicas, podrá propiciar que sus alumnos realicen actividades de aprendizaje relacionadas con el procesamiento complejo y de organización de la información como elaborar inferencias, construir categorías y redes semánticas (Pozo, 1990).

Sin duda, la estructura curricular actual, acotada por un sistema de evaluación que prioriza la memorización y el recorte de contenidos -que han de abordarse en un tiempo y en un lugar determinados, además de otros condicionantes de la cultura y organización escolar- es la primera barrera que el propio sistema establece, para propiciar el aprendizaje profundo.

Avanzar en la incorporación de dicho enfoque requiere reformular la actual estructura curricular para que, en lugar de coartar, propicie que los docentes realicen las adaptaciones curriculares que favorezcan los aprendizajes clave y significativos mediante actividades de aprendizaje no limitadas a recircular la información, sino orientadas a que el alumno analice, explique causas, relacione, formule hipótesis, entre otras.

Biggs distingue el aprendizaje profundo del superficial. En el superficial el proceso de aprendizaje se concentra en la adquisición "al pie de la letra de contenidos seleccionados"; es decir, en la "obtención de una simple lista de datos" mediante memorización selectiva. En estos casos, señala Biggs, para el alumno "el aprendizaje se convierte en una carga, una tarea que quitarse de encima" y para el profesor un problema de tiempo que le impide "dedicarse de lleno a la tarea, enfatizando la cobertura del programa a expensas de la profundidad" (2010: 34).

En el enfoque profundo, Biggs (ibid.) señala que toda actividad de aprendizaje, como el desempeño o la evidencia que se 
espera genere el alumno, han de propiciar que se desarrolle en él una necesidad de aprender.

El enfoque profundo se deriva de la necesidad sentida de abordar la tarea de forma adecuada y significativa, de manera que el estudiante trate de utilizar las actividades cognitivas más apropiadas para desarrollarla. Cuando los estudiantes sienten esta necesidad de saber, procuran centrarse en el significado subyacente: en las ideas principales, temas, principios o aplicaciones satisfactorias (ibid.: 35).

No se trata de una falsa motivación generada por un chiste o una anécdota, sino de implicar al alumno en actividades de aprendizaje que realmente se relacionen con sus necesidades e intereses.

\section{Aprendizaje significativo}

Otros enfoques expresados en el NME que se complementan con el de aprendizaje profundo son el del aprendizaje significativo y el del aprendizaje situado. El principio más relevante del aprendizaje significativo es que todo conocimiento nuevo se debe relacionar con el anterior. "el factor más importante que influye en el aprendizaje es lo que el alumno ya sabe. Averígüese esto y enséńese en consecuencia" (Ausubel, Novak y Hanesian, 1983, citado por Martí, Onrubia, 2002: 6).

Aunque este principio es fácil de entender, desarrollar en una actividad de enseñanza aprendizaje "el factor más importante", requiere habilidades específicas del profesor, entre otras, indagar cuál es ese nivel de aprendizaje que tiene el grupo de alumnos y cuáles son las concepciones alternativas que poseen, pero también necesita competencias para modificar su plan de trabajo y las estrategias de aprendizaje previamente definidas. Suponiendo que esto fuera posible, ¿¿de cuánto tiempo dispone el profesor para identificar los conocimientos previos y desarrollar la nueva estrategia de aprendizaje? Siguiendo con este enfoque, la actividad debe contener tales características que tiene que generar en el alumno un interés "para relacionar sustancial y no arbitrariamente el nuevo material con su estructura cognoscitiva” (Ausubel, 1983).

Además de esto, el enfoque propone que suceda algo que prácticamente es un sueńo en toda aula y escuela: que el alumno tenga una 
disposición para el aprendizaje. Cuando los contenidos están recortados arbitrariamente, no solo dentro de una asignatura, sino entre las disciplinas, que además tienen que memorizarse sin un significado claro para el alumno, es imposible que exista esta disposición.

La conexión entre lo más importante-los conocimientos previos-, y la capacidad de comprender de manera significativa los contenidos -el aprendizaje profundo-, se encuentra en el tercer componente del NME, en cuanto al planteamiento pedagógico; el aprendizaje situado.

\section{Aprendizaje situado}

El aprendizaje situado no solo tiene que ver con los esquemas y los conocimientos previos y con involucrar al alumno en actividades de aprendizaje orientadas a la reflexión, al análisis y al procesamiento complejo (aunque también lo hace); sino que, de manera primordial, este enfoque tiene que ver con el contexto y con la realidad en la que el alumno crece y se desenvuelve.

Soler (2006: 61) considera que el aprendizaje situado ocurre cuando la actividad cognoscitiva se da dentro de una práctica contextualizada, situada y culturalmente significativa. Esto significa que se trata de ir más allá de presentar ante un grupo "organizadores avanzados" de un tema y de involucrarlos en actividades de aprendizaje en equipo. El aprendizaje situado ocurre mediante prácticas educativas auténticas, que sean coherentes, significativas y propositivas (Díaz Barriga, 2003: 3). En este enfoque, lo nodal es que el profesor diseñe ambientes de aprendizaje o involucre a los alumnos en contextos pertinentes. Para ello recupera principios vigotskianos del aprendizaje sociocultural ya que la cognición situada parte "... de la premisa de que el conocimiento es situado, es parte y producto de la actividad, el contexto y la cultura en que se desarrolla y utiliza" (Díaz Barriga, 2003: 3). La clave es que en todas las situaciones educativas en que participan los alumnos aprendan en un contexto pertinente "se trata de una experiencia que involucra el pensamiento, la afectividad y la acción” (Baquero, 2002, citado por Díaz Barriga, 2006: 19).

El tema es cómo establecer este enfoque en los actuales contextos o culturas organizacionales de nuestras escuelas. Respecto a esto, Soler (2006: 58) señala que el aprendizaje colaborativo es la 
mejor estrategia para que los alumnos puedan participar de manera productiva en auténticas prácticas cognoscitivas compartidas.

La clave para lo "situado" es que el docente pueda crear la situación en el propio contexto escolar -o fuera de este- donde el alumno, a la vez que se involucra con sus pares, también crece y aprende mientras comparte sus conocimientos y habilidades en su propio grupo. Entre tanto, los alumnos negocian significados, intercambian información, hablan del problema a resolver, discuten y generan estrategias de solución, y es entonces cuando realmente se encuentran involucrados en actividades que originan aprendizajes profundos y significativos.

Díaz Barriga (2003: 8; 2006: 28) identifica, al menos, ocho modelos o procedimientos que propician el aprendizaje situado, favoreciendo el acercamiento a contextos reales y las actividades reflexivas, críticas, de pensamiento profundo.

1. Aprendizaje centrado en la solución de problemas auténticos.

2. Análisis de casos.

3. Método de proyectos.

4. Prácticas situadas o aprendizaje in situ en escenarios reales.

5. Aprendizaje en el servicio.

6. Trabajo en equipos cooperativos.

7. Ejercicios, demostraciones y simulaciones situadas.

8. Aprendizaje mediado por las tecnologías de la información y comunicación (TIC).

Lo importante del aprendizaje situado es que, diseñar ambientes de aprendizaje relevantes, coloca a los alumnos en una situación que los conecta con su estructura cognitiva y su acervo cultural, de manera que los conocimientos previos se activan y desempeńan su rol conforme a la actividad requerida. En estos contextos, también la disposición lógica y psicológica fluye de manera natural porque se da en un contexto social y cultural que les es familiar. Además, se da en un colectivo que está comprometido con el aprendizaje grupal.

Las estrategias auténticas y situadas pueden ser un factor que prevenga la conformación de los seudogrupos de aprendizaje, en los cuales las actividades de cada miembro se recortan y aíslan de los demás propiciando la existencia de gorrones y parásitos (Ortega y Te- 
rrazas, 2013), con el peligro de regresar al aprendizaje desvinculado, mecánico o memorístico, aun dentro del propio trabajo en equipo.

\section{Aprendizaje socioemocional}

En la Propuesta Curricular 2016 se establece, junto con los Aprendizajes Clave y la Autonomía Curricular, un tercer eje denominado Desarrollo Personal y Social. Se reconoce que para un pleno desarrollo del alumno o bien para su formación integral, necesariamente se tiene que favorecer el desarrollo de las habilidades socioemocionales, lo cual debe estar reflejado en el currículo. La justificación es que "Los aprendizajes adquieren sentido cuando verdaderamente contribuyen al pleno desarrollo personal y social de los individuos", para lo cual se incluyen las siguientes dimensiones: Apertura Intelectual, Sentido de Responsabilidad, Conocimiento de Sí Mismos y Trabajo en Equipo y Colaboración (SEP, 2016: 15-16). La competencia clave relacionada con el desarrollo de las habilidades socioemocionales de los niños y los adolescentes es "aprender a convivir" (ibíd.: 48-49), junto con "aprender a aprender". Se aclara que "un currículo de aprendizajes clave y áreas del desarrollo personal y social, como el que se plantea en este documento, dará pie a la atención debida a los procesos simultáneos de aprender a aprender y a convivir" (SEP, 2016b: 29-30). Los aprendizajes o habilidades socioemocionales que se espera que los alumnos desarrollen son:

- Autoconciencia

- Autogestión

- Conciencia social

- Habilidades para relacionarse con otros

- Toma de decisiones responsables

Para lo cual se toma como referente el programa ConstruyeT que se implementa en EMS, y que tiene como objetivo, que los alumnos logren:

- Entender y manejar las emociones.

- Establecer y alcanzar metas positivas. 
- Sentir y mostrar empatía hacia los demás.

- Establecer y mantener relaciones colaborativas (SEP, 2016b: 29-30).

Tanto los aprendizajes socioemocionales que propone la PC como los objetivos planteados en el programa ConstruyeT son recuperados del Collaborative for Academic, Social and Emotional Learning (CASEL; www.casel.org), un organismo de prestigio internacional que promueve el desarrollo de programas escolares para la formación socioemocional.

En el plan de estudios 2011, de alguna manera ya están expresadas las competencias socioemocionales, las cuales se dividen en competencias para la convivencia y competencias para la vida en sociedad. Las primeras incluyen empatía, relacionarse armónicamente con otros y la naturaleza, ser asertivo, trabajar de manera colaborativa, tomar acuerdos y negociar con otro; y las segundas, decidir y actuar con juicio crítico frente a los valores y las normas sociales y culturales, proceder en favor de la democracia, la libertad, la paz, el respeto a la legalidad y a los derechos humanos (SEP, 2011a: 38).

En el mapa curricular de la PC se expresan como: Desarrollo personal y para la convivencia, y Desarrollo personal y social (SEP, 2016b: 41).

La relevancia de favorecer el desarrollo del aprendizaje socioemocional es que, además del valor intrínseco que posee para el desarrollo integral del individuo, los estudios muestran que tiene un efecto directo en el éxito académico (Repetto, 2009: 12).

El desarrollo de las competencias socioemocionales también previene factores de riesgo en el aula, comportamientos agresivos y antisociales; por otra parte, tienen implicaciones en la mejora de las calificaciones y el desempeño escolar, además de que se favorecen los comportamientos prosociales, los cuales, a su vez, se relacionan con resultados intelectuales y académicos positivos (Berger, et al., 2014).

El establecimiento de programas orientados a favorecer el aprendizaje socioemocional ha demostrado en estudios meta-analíticos que "los estudiantes que participaron de dichos programas aumentaron sus competencias socioemocionales, sus actitudes y conductas prosociales, y subieron en 11 puntos porcentuales su rendimiento académico" (Milicic, et al., 2013: 646). Para el caso de México, es importante evaluar y recuperar la experiencia de 
ConstruyeT para revisar la pertinencia de su implementación en educación básica.

\section{EJES DE POLÍTICA}

\section{La Escuela al Centro}

Indudablemente, los enfoques del aprendizaje profundo, significativo y situado juegan un papel preponderante en el NME, porque se enlazan de manera importante con los pronunciamientos más amplios de política educativa, como lo son el de "la escuela al centro", el de "equidad con inclusión", así como el del "sistema de apoyo técnico a la escuela”.

La escuela al centro se describe como una "[...] escuela renovada y fortalecida, que cuente con una organización, recursos, acompańamiento, infraestructura, docentes y servicios que conviertan las aulas en auténticos espacios de aprendizaje" (SEP, 2016a: 22). Las Reglas de Operación del Programa para el Desarrollo Profesional Docente establecen que, lo que se busca con este modelo “[...] es reconstruir la capacidad de la escuela pública de Educación Básica para definir sus propias estrategias en un marco de corresponsabilidad, de seguimiento y asistencia técnica especializada, espacio en donde convergen todos los recursos y esfuerzos de los distintos actores" (citado por SEP, 2016a: 22).

El NME pretende desarrollar "[...] mecanismos institucionales que permitan a las autoridades educativas conocer y atender, con oportunidad y pertinencia, las necesidades de las escuelas" (SEP 2016a: 35), a la vez que "[...] permite a maestros, directores, supervisores y otras autoridades desarrollar soluciones a sus problemas educativos con mayor autonomía y creatividad”.

La Propuesta Curricular 2016, al mismo tiempo que procura dotar de "mayor autonomía de gestión", plantea una estrategia de autonomía curricular, junto con otros dos componentes: los aprendizajes clave y el desarrollo personal social (ver Mapa 1). El mismo documento establece ocho condiciones para gestionar el nuevo currículo en el marco de dicha autonomía.

1. Liderazgo directivo.

2. Ética del cuidado. 
3. Infraestructura y equipamiento.

4. Materiales educativos.

5. Servicio de asistencia técnica a la escuela (SATE).

6. Evaluación de los aprendizajes.

7. Formación continua de maestros.

La autonomía escolar, en general, se entiende como "la transferencia de responsabilidades desde un nivel de gobierno superior hacia la escuela, acompañada de los recursos necesarios para su ejecución. Los recursos pueden ser en dinero, equipamiento, material didáctico o las condiciones legales que permitan a la escuela cumplir con las nuevas funciones que se le transfieren" (Espínola, 2000: 3).

A pesar de que la PC no desarrolla conceptualmente el término "autonomía curricular", señala que es de observancia nacional, para lo cual "cada escuela determinará los contenidos programáticos del componente, con base en las horas lectivas que tenga disponibles y en los lineamientos que expida la SEP para normar los espacios curriculares de la Autonomía curricular" (SEP, 2016b: 83).

Los ámbitos donde se organiza la autonomía curricular, de acuerdo con el porcentaje otorgado al nivel (preescolar: de 15 a 50\%; primaria: de 11 a 43\%; y secundaria: de 14 a 33\%.) son:

1. Profundización de aprendizajes clave.

2. Ampliación del desarrollo personal y social.

3. Nuevos contenidos relevantes.

4. Contenidos regionales y locales.

5. Impulso a proyectos de impacto social.

La autonomía curricular tiene una tradición de décadas en países de Sudamérica y Centroamérica (principalmente El Salvador y Honduras), así como en países europeos, no siempre con resultados favorables en el aprovechamiento de los alumnos. En un estudio realizado por Calero (2009) en España, en el que analiza los resultados de PISA en función del grado o tipo de autonomía de los planteles escolares, encontró que los centros que no tienen autonomía puntúan más que los centros que sí la tienen. No obstante, encontró que si el sistema tiene autonomía, "aunque la escuela evaluada en concreto no la tenga, los alumnos 
puntúan mejor que si el sistema no tuviera autonomía”, no así cuando la autonomía es en el uso de presupuesto y de contratación de profesores. Finalmente, reconoce que en los resultados existe un grado de complejidad en el efecto de la autonomía sobre la puntuación de PISA.

\section{MAPA 1. Nuevo Modelo Educativo, 2016}

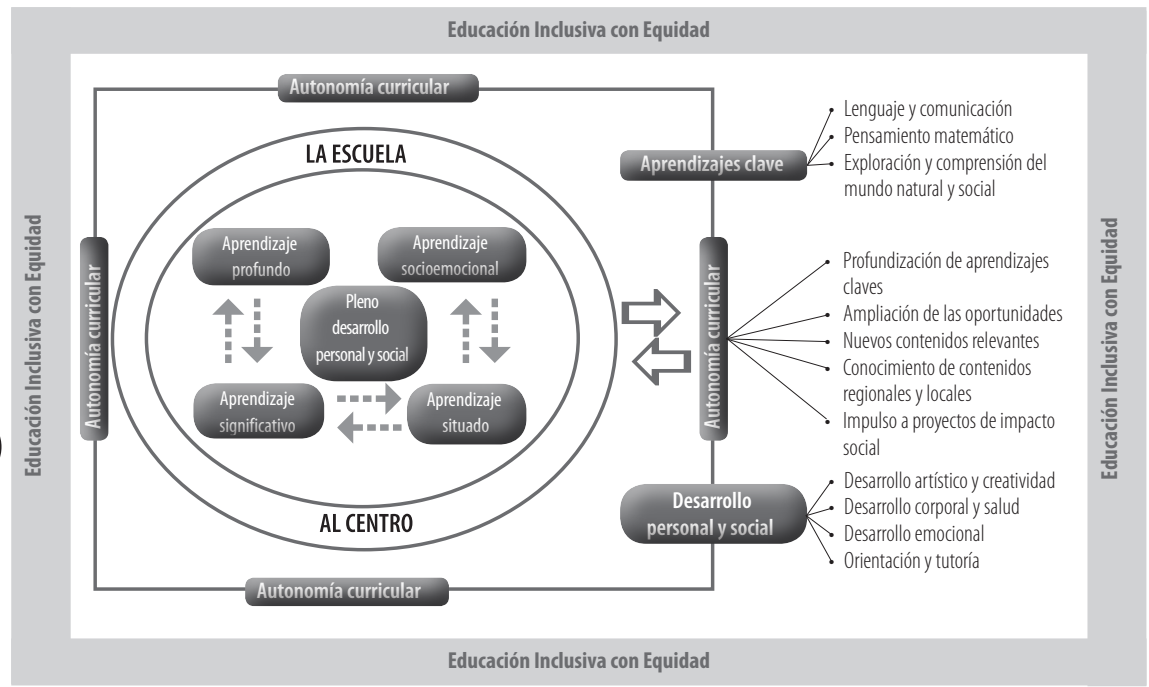

Fuente: Elaboración propia con base en SEP, 2016.

Elena Martín considera que se deben cumplir al menos tres requisitos para proporcionar autonomía curricular en las escuelas: a) a mayor autonomía, mayor evaluación y mayor control; b) establecer otros niveles de responsabilidad, horizontales y verticales, con el fin de alcanzar un grado óptimo de unidades de vertebración, y c) a mayor autonomía, mayor formación del profesorado. Insiste en que establecer autonomía curricular sin considerar estos principios, además de que existen riesgos, es una actitud "irresponsable y capaz de generar todo tipo de desigualdades y conflictos" (2009: 152-153). En este sentido, en un estudio realizado en Argentina sobre los efectos de la autonomía escolar, se concluye que no siempre constituye un proceso deseable y posible y que, más allá de liberar a los docentes de trabajo excesivo, los sobrecarga con exigencias que les resultan desmedidas (Astiz, 2015). 


\section{Educación inclusiva con equidad}

Constituir una escuela renovada y fortalecida es un verdadero reto en nuestro país, ya que las brechas de insumos, procesos y resultados entre escuelas pobres y ricas son realmente dramáticas. Si la política educativa nacional solo se enfocara en resolver esta situación, se estaría dando un salto cualitativo y cuantitativo no solo en la mejora de la calidad de la educación, sino además se estarían atendiendo principios de justicia social.

De acuerdo con Echeita y Ainscow (2010: 29) el "[...] objetivo final de la educación inclusiva es contribuir a eliminar la exclusión social que resulta de las actitudes y las respuestas a la diversidad racial, la clase social, la etnicidad, la religión, el género o las aptitudes entre otras posibles". El enfoque parte de "la convicción que la educación es un derecho humano fundamental y el cimiento de una sociedad más justa. En este sentido, es una manera de asegurar que la Educación Para Todos signifique realmente todos" (UNESCO, 2008: 6) y pone particular énfasis en aquellos grupos de alumnos que están en situación de marginación, exclusión, o fracaso escolar.

Según el Modelo de Atención de los Servicios de Educación Especial (SEP, 2011: 10), la adhesión de México a la Convención sobre los Derechos de las Personas con Discapacidad y a su Protocolo Facultativo, aprobados en 2006, lo compromete, de forma vinculante "[...] a reconocer el derecho de las personas con discapacidad a la educación, sin discriminación y sobre la base de la igualdad de oportunidades. Al mismo tiempo, compromete a asegurar un sistema de educación inclusiva a todos los niveles y a lo largo de la vida". En función de este compromiso, el programa sectorial de educación 2013-2018 establece, entre sus objetivos "Asegurar mayor cobertura, inclusión y equidad educativa entre todos los grupos de la población para la construcción de una sociedad más justa”, estableciendo líneas de acción para desarrollar "estrategias metodológicas y materiales educativos apropiados para la atención de los diversos tipos de discapacidad o de problemas para el aprendizaje" y promover "modelos y prácticas escolares que permitan la inclusión en todos los niveles educativos" (SEP, 2013: 54-57).

En complemento con el plan sectorial, el NME retoma esta demanda e incorpora la propuesta de eliminar las barreras de apren- 
dizaje y postula "[...] la eliminación de las barreras que impiden el aprendizaje y la participación a todos los alumnos, con especial énfasis en aquellos que presentan condiciones de vulnerabilidad" (2016: 17). En suma, se trata de que las escuelas para pobres ya no sean factor de reproducción social, y que los alumnos provenientes de familias con menor capital cultural y económico, permanezcan en ellas, avancen en el tiempo estipulado y adquieran los conocimientos relevantes para la vida, a fin de garantizar que todos logren los aprendizajes esperados, independientemente de su condición de origen.

Eliminar las barreas de aprendizaje implica caracterizar las condiciones de la oferta educativa, incluyendo la ubicación de la escuela, las características de la infraestructura, las expectativas docentes, la cultura y el clima escolar que estorban que el aprendizaje significativo y pertinente se lleve a cabo.

Introducir el concepto de barreras de aprendizaje a todo el sistema educativo, y no solo a los planteles y al personal que atiende a niños con NEE o sobresalientes, favorece no solo la revisión de un concepto más amplio, como el de educación inclusiva con equidad, sino obliga a un análisis más detenido de la educación como un derecho (Tomasevski, 2004).

Muchas veces las barreras de aprendizaje se encuentran en las propias condiciones de la oferta educativa en cuanto a infraestructura se refiere. La Evaluación de las Condiciones de Aprendizaje (Ahuja, 2016: 29) realizada por el INEE informa con detalle en qué condiciones se encuentran las escuelas públicas en México: solo $42.5 \%$ de ellas proporciona a sus estudiantes agua purificada para beber; en $13.7 \%$ hay menos aulas para la cantidad de grupos; $31 \%$ considera que el aula es pequeña para el número de estudiantes; solo en $43.1 \%$ de escuelas los estudiantes tienen acceso a, por lo menos, una computadora y en $56.9 \%$ no hay Internet.

Con respecto a las condiciones que aseguren la inclusión de niños con algún tipo de discapacidad, en promedio, carecen de puertas amplias para acceso a silla de ruedas o con muletas $(27.5 \%)$, rampas útiles para el acceso y la circulación (24.8\%), sanitarios amplios y con agarraderas (10.4\%).

Una comparación sobre la escasez de los recursos materiales de las escuelas secundarias entre México y los países TALIS muestra grandes diferencias en todos los rubros, siempre en favor de los últimos. La proporción de docentes que trabaja en escuelas 
que carecen de materiales didácticos es de $44 \%$ en México y $26 \%$ en los países TALIS; de materiales para biblioteca, 56 y 29\%; de equipos de cómputo, 71 y 38\%; acceso a Internet, 71 y 30\%, y programas informáticos, 70 y $38 \%$ respectivamente (Backhoff y Pérez-Morán, coords., 2015: 32).

\section{CONCLUSIONES}

El propósito del NME de incorporar el aprendizaje profundo y situado de acuerdo con su planteamiento pedagógico, es que los alumnos lleguen a desarrollar esquemas de pensamiento complejo y aprendizajes clave. Se reconoce que, para favorecer dicho pensamiento, así como "los conocimientos, las competencias y los valores plasmados en el perfil de egreso del estudiante" (SEP, 2016: 38) es imprescindible la revisión del plan de estudios para "superar la saturación de contenidos y seleccionarlos de manera cuidadosa". En este esquema, "la organización tradicional" de los conocimientos ya no resulta pertinente para favorecer el desarrollo de competencias para la vida. De acuerdo con el NME la función de la escuela, "ya no es enseñar" (ibid.: 41), sino favorecer que los alumnos aprendan a aprender. En consecuencia, recoge el planteamiento de la UNESCO, señalando que los alumnos no solo habrán de desarrollar estas competencias metacognitivas, sino que también aprenderán a convivir, aprenderán a ser y, en el caso de EMS, aprenderán a hacer.

En este sentido el NME es congruente en su planteamiento; es necesario un rediseño curricular y establecer nuevas formas de trabajo en el aula que favorezcan -más que el recorte y repaso de contenidos aislados-, el desarrollo de competencias y aprendizajes clave. Como acertadamente establece Biggs (2010: 35) "[...] el primer paso para mejorar la enseñanza consiste en evitar los factores que estimulan un enfoque superficial".

Es innegable que la autoridad educativa deberá hacer un análisis exhaustivo para identificar y remover todas aquellas variables que mantienen por tiempo indefinido prácticas docentes anquilosadas que propician el aprendizaje memorístico y aislado de hechos y conceptos. Habrá que revisar en qué medida los actuales procedimientos de dosificación de contenidos y el sistema de calificaciones (Anderson, 2016) que impone la autoridad educativa local, funcionan como barrera para el aprendizaje profundo y situado. El 
mayor problema de ese sistema de calificaciones es que obliga a los profesores a abordar con los alumnos una gran dosis de contenidos de manera superficial con el fin de que "aprueben" el examen.

También es importante analizar qué tanto de la carga administrativa, la estructura organizacional de la escuela, las exigencias del tipo de plaza o contrato, el tamaño del grupo, la infraestructura, entre otros, están determinando que la educación en México esté anclada a formas y estilos que ya no corresponden a la realidad de los alumnos y los retos que demanda los procesos de enseñanza aprendizaje activos.

Un modelo de autonomía como el que presenta la propuesta curricular implica nuevas responsabilidades y competencias tanto para funcionarios como para docentes y directores escolares. Por esta razón, en el contexto educativo de nuestro país, impulsar dicha autonomía, puede generar un retroceso en la mejora educativa; sobre todo si lo valoramos a la luz de las dificultades que se han presentado para implantar otras medidas menos complicadas como son el servicio de apoyo técnico a la escuela (SATE) y el programa de tutorías, dos políticas centrales de la Reforma Educativa.

Para implementar la propuesta de autonomía curricular, tanto directivos como docentes, deberán ser competentes en el diseño curricular, la planeación didáctica de acuerdo con los enfoques aquí analizados, contar con un amplio repertorio de estrategias y técnicas de aprendizaje acordes con el NME, así como en la realización de adecuaciones curriculares conforme a criterios de integración e inclusión educativa.

Ante la ausencia de esto, una solución puede ser que se implementen esquemas paulatinos de autonomía curricular, de tal manera que solo los directivos que han demostrado dichas competencias, puedan incorporar en su escuela el modelo de autonomía que se propone; para ello, los centros de formación docente habrán de desarrollar estrategias de capacitación de tal manera que los egresados cuenten con un "certificado en autonomía curricular", por ejemplo.

Por otra parte, dadas, las condiciones de la oferta educativa en el país, la premisa de una educación inclusiva con equidad necesariamente debe estar orientada a "[...] contrarrestar la desigualdad de insumos para los pobres igualando el gasto por alumno en distintas escuelas y contextos", de otra manera, se continuará ofertando una educación de segunda para los pobres, perpetuan- 
do el ciclo intergeneracional de pobreza ya que "... las carencias del hogar y la escuela suelen reforzarse, y explican las grandes diferencias de los resultados" (Reimers, F., 2000: 17).

Conviene rescatar el principio de "proporcionalidad solidaria” definido por Pablo Latapí (1993) -que plantea que el reparto de los recursos debe procurar el fortalecimiento de los más débiles, igualando y compensando insumos-. Vale la pena ponerlo sobre la mesa porque las brechas y la desigualdad del sistema educativo mexicano son tan grandes, que ningún esfuerzo aislado de los profesores, directivos y autoridades locales puede solucionar el problema. La educación inclusiva con equidad requiere el esfuerzo y el compromiso de todos; sociedad civil, empresarios, gobierno, legisladores. Es urgente una verdadera proporcionalidad solidaria, no solo como planteaba Latapí (1993), destinando recursos de educación superior a la educación básica, sino mayor aún, destinando recursos de áreas donde el derroche y el uso para fines menos prioritarios consumen grandes partidas presupuestarias. Es hora de que realmente la sociedad ponga a la educación, a la escuela, al centro.

\section{REFERENCIAS BIBLIOGRÁFICAS}

Ahuja, Raquel. Evaluación de condiciones básicas para la enseñanza y el aprendizaje (ECEA). Primaria, México, INEE, 2016. Disponible en: http://www.inee.edu.mx/index.php/publicaciones-micrositio Fecha de consulta, 15 de junio de 2016.

Astiz, M. "Autonomía educativa en la provincia de Buenos Aires, Argentina: comparación entre dos distritos escolares. Una perspectiva neo-institucionalista”, en Educação \& Sociedade, 36 (julio-septiembre), 2015. Disponible en: http://148.215.1.176/articulo.oa?id=87342690004>ISSN 0101-7330. Fecha de consulta, 10 de septiembre de 2016. Ausubel, D. Novak, J, Hanesian, H. Psicologia Educativa: Un punto de vista cognoscitivo, México, Trillas, 1983.

Backhoff, E., y Pérez-Morán, J. C. (Coords.). Segundo Estudio Internacional sobre la Enseñanza y el Aprendizaje (TALIS 2013). Resultados de México, México, INEE, 2015. Disponible en www.inee.edu.mx/images/stories/2015/mesaTalis/.../TALIS_2013_web2.pdf. Fecha de consulta, 15 de agosto de 2016. 
Biggs, J. Calidad del aprendizaje universitario, Madrid, Narcea, 2010. Berger, C., Milicic, N., Alcalay, L., C. y Torreti, A. "Programa para el Bienestar y Aprendizaje Socioemocional en estudiantes de tercero y cuarto grado: descripción y evaluación de impacto", en Revista Latinoamericana de Psicología, 46(3), 2014, pp. 169-177. Disponible en: http://www.elsevier.es/es-revista-revista-latinoamericana-psicologia-205-articulo-programa-elbienestar-aprendizaje-socioemocional-S0120053414700202. Fecha de consulta, 10 de agosto de 2016.

Calero, J. "Resultados educativos y autonomía. Un análisis basado en PISA 2006", en La autonomía de los centros educativos. VI Encuentros sobre educación en El Escorial (UCM), Comunidad de Madrid, 2009, pp. 135-150. Disponible en www.madrid.org/bvirtual/BVCM001907.pdf . Fecha de consulta, 23 de septiembre de 2016.

Díaz Barriga, F. "Cognición situada y estrategias para el aprendizaje significativo", en Revista Electrónica de Investigación Educativa, 5(2), 2003. Disponible en http://redie.ens. uabc.mx/vol5no2/contenido-arceo.html. Fecha de consulta, 10 de septiembre de 2016.

Díaz Barriga, F. Enseñanza situada. Vinculo entre la escuela y la vida, México, McGraw Hill, 2006.

Echeita, G. Ainscow, M. "La Educación inclusiva como derecho. Marco de referencia y pautas de acción para el desarrollo de una revolución pendiente", en Tejuelo: Didáctica de la Lengua y la Literatura. Educación, 12, 2010, pp. 26-46. Disponible en https://dialnet.unirioja.es/servlet/articulo?codigo $=3736956$. Fecha de consulta, 30 de septiembre de 2016.

Espínola, V. Autonomía escolar: factores que contribuyen a una escuela más efectiva, BID, 2000. Disponible en: http:// www.iadb.org/wmsfiles/products/publications/documents/1017850.pdf Fecha de consulta, 11 de agosto de 2016.

INEE. Evaluación de condiciones básicas para la enseñanza y el aprendizaje (ECEA) primaria, Ciudad de México, 2016. Disponible en http://www.inee.edu.mx/index.php/publicaciones-micrositio. Fecha de consulta, 11 de septiembre de 2016.

Latapí, P. "Reflexiones sobre la justicia en la educación", en Revista Latinoamericana de Estudios Educativos, vol. XXIII, núm. 2, México, CEE, 1993, pp. 9-41, 
López, A. y López, A. "Los enfoques de aprendizaje. Revisión conceptual y de investigación", en Revista Colombiana de Educación, 64 (1), Bogotá, Colombia, 2013. Disponible en: www.scielo.org.co/pdf/rcde/n64/n64a06.pdf. Fecha de consulta, 11 de septiembre de 2016.

Martí, S. y Onrubia, J. Las teorías del aprendizaje escolar, España, Editorial UOC, Universidad Virtual, 2002.

Milicic, N., Alcalay, L., Berger, C. y Álamos, P. "Aprendizaje socioemocional en estudiantes de quinto y sexto grado: presentación y evaluación de impacto del programa BASE", en Ensaio: Avaliação e Políticas Públicas em Educação, 21 (octubre-diciembre), 2013. Disponible en http://www. redalyc.org/articulo.oa?id=399538147002. Fecha de consulta, 18 de agosto de 2016.

Martín, E. "Autonomía escolar y evaluación: dos pilares de la calidad de la enseñanza", en La autonomía de los centros educativos. VI Encuentros sobre educación en el escorial (UCM), Comunidad de Madrid, 2009, pp 151-162. Disponible en www.madrid.org/bvirtual/BVCM001907.pdf. Fecha de consulta, 11 de septiembre de 2016.

Ortega, F., Terrazas, V. De los seudogrupos a los grupos de aprendizaje cooperativo. Primer Congreso Internacional de Educación, Universidad Autónoma de Chihuahua. Facultad de Filosofía y Letras, México, 2013. Disponible en cie.uach.mx/cd/docs/ area_01/a1p12.pdf. Fecha de consulta, 12 de mayo de 2016,

Pozo, J. I. "Estrategias de aprendizaje", en C. Coll, J. Palacios, A. Marchesi. Desarrollo psicológico y educación II. Psicología de la Educación, (2), Madrid, Alianza, 1990, pp. 199-221.

Reimers, F. "¿Pueden aprender los hijos de los pobres en las escuelas de América Latina?", en Revista Mexicana de Investigación Educativa, 5(9), México, 2000, pp 11-69. Disponible en www.comie.org.mx/documentos/rmie/ v05/n009/pdf/rmiev05n09scC00n01es.pdf. Fecha de consulta, 12 de octubre de 2016.

Repetto, E. (Dir). Formación en competencias socioemocionales. $L i$ bro del Formador, Madrid, La Muralla, 2009.

SEP Plan de estudios 2011. México, 2011a. Disponible en http://www.curriculobasica.sep.gob.mx/. Fecha de consulta, 2 de septiembre de 2016. 
SEP Modelo de atención de los servicios de educación especial 2011, México, Dirección de Educación Especial, 2011 b. Disponible en educacionespecial.sepdf.gob.mx/documentos/MASEE2011.pdf . Fecha de consulta 12 de agosto de 2016

SEP Programa Sectorial de Educación 2013-2018, México, SEP, 2013. Disponible en http://www.sep.gob.mx/es/sep1/ programa_sectorial_de_educacion_13_18\#.WC4VYiQwBQM. Fecha de consulta, 30 de julio de 2016,

SEP El Modelo educativo 2016. El planteamiento pedagógico de la Reforma Educativa, México, SEP, 2016a. Disponible en https:/www.gob.mx/cms/uploads/attachment/file/.../ Modelo_Educativo_2016.pdf. Fecha de consulta, 30 de julio de 2016.

SEP Propuesta curricular para la educación obligatoria, 2016. México, SEP, 2016b. Disponibe en https://www.gob.mx/ cms/uploads/docs/Propuesta-Curricular-baja.pdf. Fecha de consulta, 30 de julio de 2016.

Soler, E. Constructivismo, Innovación y Enseñanza Efectiva, Caracas, Equinoccio, 2006.

Subsecretaría de Educación Media Superior. "Programa ConstruyeT 2014-2018. Fortalecer las capacidades de la escuela para promover el desarrollo integral de los jóvenes", México, SEP, (s/f). Disponible en: www.construye-t.org. $\mathrm{mx} /$ resources/DocumentoConstruyeT.pdf. Fecha de consulta, 5 de septiembre de 2016.

UNESCO. "La educación inclusiva: el camino hacia el futuro", en Cuadragésima octava reunión, Centro Internacional de Conferencias, Ginebra, 25 a 28 de noviembre de 2008. Ginebra, Suiza. Disponible en: http://www.ibe.unesco. org/fileadmin/user_upload/Policy_Dialogue/48th_ICE/ CONFINTED_48-3_Spanish.pdf. Fecha de consulta, 12 de septiembre de 2016,

Tomasevski, K. "Indicadores del derecho a la educación", en Revista IIDH, México, 40, 2004, pp 341-388. Disponible en: www.juridicas.unam.mx/publica/librev/rev/iidh/ cont/40/pr/pr18.pdf. Fecha de consulta, 5 de septiembre de 2016. 